\title{
Chapter 65. Occupational exposure to Aflatoxin B1 in Portuguese swine farms
}

S . Viegas, L . Veiga , P . Figueredo, A . Almeida, E . Carolino and C . Viegas

Citation Information

Occupational Safety and Hygiene

Edited by Gonçalo Perestrelo

CRC Press 2013

Pages 373-376

Print ISBN: 978-1-138-00047-6

eBook ISBN: 978-0-203-72965-6

DOI: $10.1201 / \mathrm{b} 14391-78$ 


\title{
Occupational exposure to Aflatoxin $\mathrm{B}_{1}$ in Portuguese swine farms
}

\author{
S. Viegas, L. Veiga, P. Figueredo, A. Almeida, E. Carolino \& C. Viegas \\ ESTeSL, IPL, Lisbon, Portugal
}

\begin{abstract}
In 1987, the International Agency for Research on Cancer concluded that there was sufficient evidence for carcinogenicity of naturally occurring aflatoxins in humans. Regarding occupational exposure to this chemical agent, farmers and other agricultural workers present a higher risk due to airborne aflatoxin via inhalation of dust. This study was carried out in 7 swine farms located at the district of Lisbon, Portugal. Blood samples were collected from a total of 11 workers. In addition, a control group $(\mathrm{n}=25)$ was included that conducted administrative tasks in an educational institution without any type of agricultural activity. Results obtained suggest that occupational exposure to $\mathrm{AFB}_{1}$ by inhalation occurs and represents an additional risk in this occupational setting that need to be recognized, assessed and, most important, prevented.
\end{abstract}

\section{INTRODUCTION}

Aflatoxins were first isolated about 40 years ago after outbreaks of disease and death in turkeys (Williams et al., 2004) and cancer in rainbow trout (Rucker et al., 2002; Williams et al., 2004) fed with rations formulated from peanut and cottonseed meals. These toxins are secondary metabolites produced under certain conditions of temperature, $\mathrm{pH}$ and humidity predominantly by Aspergillus flavus and Aspergillus parasiticus fungi species (Bhatnagar et al., 2006).

Among 18 different types of aflatoxins identified, major members are aflatoxin $B_{1}, B_{2}, G_{1}$ and $\mathrm{G}_{2}$. Aflatoxin $\mathrm{B}_{1}\left(A F \mathrm{~B}_{1}\right)$ is normally predominant in cultures as well as in food products. $\mathrm{AFB}_{1}$ was shown to be genotoxic and a potent hepatocarcinogen (IARC, 1993; Dash et al., 2007). This mycotoxin is metabolized by the mixed function oxidase system to a number of hydroxylated metabolites including the 8,9-epoxide. The latter is considered to be the ultimate carcinogen that reacts with cellular deoxyribonucleic acid (DNA) and proteins to form covalent adducts (Autrup et al., 1991; Richard, 1998; Brera et al., 2002; Dash et al., 2007).

In 1987, the International Agency for Research on Cancer concluded that there was sufficient evidence for carcinogenicity of naturally occurring aflatoxins in humans (IARC, 1987). This conclusion was reaffirmed in two subsequent re-evaluations (IARC 1993, 2002), based upon results from several cohort studies in China and Taiwan that reported associations between biomarkers for aflatoxin exposure and primary liver-cell cancer.

Occupational exposure to this mycotoxin may occur by inhalation of dust generated during the handling and processing of contaminated crops and feeds. Therefore, farmers and other agricultural workers present a higher risk for occupational exposure due to airborne aflatoxin via inhalation of dust (Flannigan and Gillian, 1996; Ghosh et al., 1997; Brera et al., 2002).

To confirm exposure, mycotoxins and/or mycotoxin metabolites may be detected in biological samples using biomarkers (Hooper et al., 2008).

Swine production is known to be an occupational setting that involves high occupational exposure to particulate matter and fungi (Donham et al., 1989; Vogelzang et al., 2000; Duchaine et al., 2000; Kim et al., 2007; Kim et al., 2008). Thus it is conceivable that swine production workers are exposed via inhalation to aflatoxins. The aim of this study was to determine whether swine workers in Portugal were exposed to aflatoxin $\left(\mathrm{AFB}_{1}\right)$.

\section{MATERIALS AND METHODS}

This study was carried out in 7 swine farms located at the district of Lisbon, Portugal, between January and May 2011. The pig buildings investigated in this research were all classified as the manure removal system where manure can be removed from the pig building completely several times a day. In some swine's places, such as in the maternity and where the males were confine, the floor is cover with straw or journal paper.

The ventilation modes of the pig buildings were mechanical ventilation by wall exhaust fans and natural ventilation by operation of a winch-curtain.

Blood samples were collected from a total of 11 workers. In addition, a control group $(\mathrm{n}=25)$ was included that conducted administrative tasks in an 